\title{
Biomechanical Analysis of Achilles Tendon Stretch And Plantar Fascia Stretch In Plantar Fasciitis Treatment
}

\author{
Jie HU, Dong-Mei WANGa,, ${ }^{a}$, Wen-Ting YANG, Qin-Yang GUO and Guang-Lin \\ SHI
}

School of Mechanical Engineering, Shanghai Jiao Tong University, 200240 Shanghai, China

Keywords: Achilles Stretch, Plantar Stretch, Plantar Fasciitis, Biomechanics, Simulation.

\begin{abstract}
This paper aims to simulate two stretching methods adopted in plantar fasciitis treatment in MSC.ADAMS, Achilles tendon stretch and plantar fascia stretch. Clinical evidence has been accumulated that stretching is effective in relieving heel pain caused by plantar fasciitis. While plantar fascia stretching is conceived to be more effective than the traditional Achilles tendon stretch, the reason leading to this difference has not been identified yet. A multi-segment foot model consisted of ligaments, plantar fascia, plantar soft tissue was constructed and then validated by comparing the ground reaction forces and joint rotation angles obtained in a gait simulation with previous research results. The model was then applied in finding out the root cause in these two stretching methods by analysing the deformation of plantar fascia. Achilles tendon stretch was performed to collect kinematic data of tibia using NDI, which was used to drive the simulation. In the plantar fascia stretch, a rotational plate was placed beneath the fore-foot and had a range of motion from $0^{\circ}$ to $20^{\circ}$. The forefoot was supported and lifted to simulate the stretching process. Deformation of the plantar fascia under two stretching methods were compared. The result shows that average deformation in Achilles tendon is smaller than that of plantar fascia stretch which may cause the therapeutic difference between these two stretch methods. This analysis method could also be applied in refining physical therapy treatment.
\end{abstract}

\section{Introduction}

Plantar fascia stretches from the calcaneal tubercle to the metatarsal heads and proximal phalanx of each toe, which enable them to stable human foot arch in standing and walking [1]. High intensity of exercise or repetitive over stretching could cause micro damages to plantar fascia, so plantar fasciitis is widely spread, causing great pain and inconvenience. Researchers have found that there is no inflammation in plantar fascia [2]. Lemont used ultrasound to obtain the sonographic image of plantar fascia and found that there was no significant changes in plantar fascia's thickness in patients [3]. Various treatments tend to be ineffective or takes long time to mitigate the pain. Conservative treatments including physical therapy, orthoses insoles, night splints and foot bath are usually adopted firstly. Operative methods would be taken if these mild ways do not work, such as injection of steroid, plantar fascia release etc. and ultrasound shock wave therapy has also been widely used [4-7]. Plantar fascia release has been proved to have negative effect on bony structure and exert extra tension on remaining plantar fascia and ligamentous structure [8-9]. Injection of steroid provides a fast pain relief but the effect has been reported not long-lasting [10]. So researches concerning improving conservative methods is worthy.

Among the commonly used conservative treatments, stretching has been widely adopted in the early stage of plantar fasciitis to recover the stiffness and elongation ability of plantar fascia. Stretch therapy includes following types: with or without muscle strengthening, plantar fascia stretch and Achilles tendon (AT) stretch [11-13]. Patients' condition determines whether to use single stretch method or combine them together. Since patients with plantar fasciitis always have a tight Achilles tendon, Achilles tendon stretch is used to decrease its tension and increase that of plantar fascia [14]. Plantar fascia stretch is operated directly on phalanges to tighten plantar fascia so to increase the metabolic rate and relief the pain [12]. There is clinical evidence that Achilles tendon stretch is less effective than plantar fascia stretch but the mechanism that leads to this difference has not been clarified yet [15]. 
Meanwhile, computational foot model has become an important tool in understanding foot and ankle aetiologies since various diseases can be simulated by changing the features of the model [16]. One multi-segment dynamic model was developed and can be used in understanding why plantar fascia stretch has better clinical effects than Achilles tendon stretch has by simulating these two therapies and analysing the results in biomechanical perspective.

\section{Materials and Methods}

\section{Multi-Segment foot Model Validation}

The model used in this paper was composed of fibula, tibia and 26 bones of foot. Bone models were obtained from previous researches of our research group [17]. In recent researches, foot has been widely modelled as multiple segments while the number of the segments range from 2 to 4 [18-22]. This paper has divided the foot into 4 parts: rear-foot composed of calcaneus and talus, mid-foot composed of navicular, cuneiforms, cuboid, fore-mid foot consisted of first to fifth metatarsal, fore-foot composed of phalanges. Tibia and fibula were connected with rear-foot using a spherical joint while the connections between rear-foot and mid-foot, mid-foot and fore-mid foot, fore-mid foot and fore-foot were all rotational joints [23]. The defined three-dimensional model was as shown in the Fig.1.

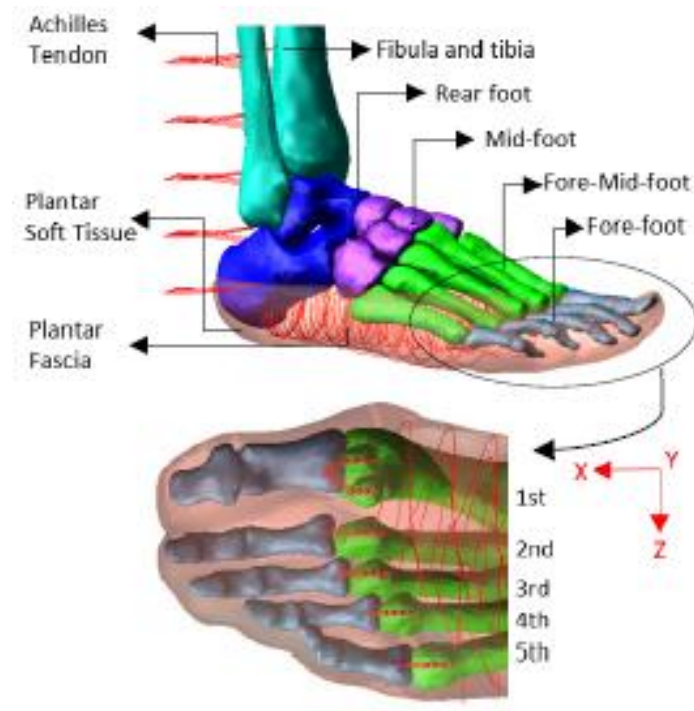

Figure 1. Multi-segment Foot Model

Fascia and ligaments were modelled using spring-dampers and their geometries were based on anatomical location on bones. Plantar fascia have been modelled as five rays corresponding to the order of five metatarsal. One special structure in plantar fascia model were four spheres locating beneath each metatarsal head which had contact with metatarsal so to simulate the interaction of plantar fascia with metatarsal in vivo [24]. Stiffness and damping ratio were defined according to previous researches [25]. In this paper, Achilles tendon was modelled using spring damper and was attached on the calcaneus directly and relevant material parameters were as following [25].

Table 1. Material properties

\begin{tabular}{|l|l|l|}
\hline Element & Stiffness/( N·mm-1) & Damping/(N·s· mm-1) \\
\hline Ligament & 50 & 0.3 \\
\hline Fascia & 8 & 0.3 \\
\hline Achilles Tendon & 15 & 0.3 \\
\hline
\end{tabular}

Another critical component is plantar soft tissue. According to Erdemir [26], plantar soft tissue can be regarded as linear elastic when its deformation is smaller than $35 \%$ and Adams/Flex 
provides an easy access to flexible body that allows big elastic deformation. Thus, plantar soft tissue was obtained using this module. Plantar soft tissue model was imported into Abaqus to obtain modal neutral file which was then imported into ADAMS. Elasticity modulus was set as $0.91 \mathrm{MPa}$, Poisson ratio was 0.49 [27].

One stance phase of gait was simulated to validate this foot model. Choosing the top point of tibia as the control point. The distance from sole to the control point was $400 \mathrm{~mm}$. Data used in this simulation was from the gait experiment of Salford University, including the displacement and rotational data, forces and torques at control point that were calculated through inverse dynamics as were shown in Fig. 2 [28]. The whole simulation process lasted $0.7 \mathrm{~s}$.

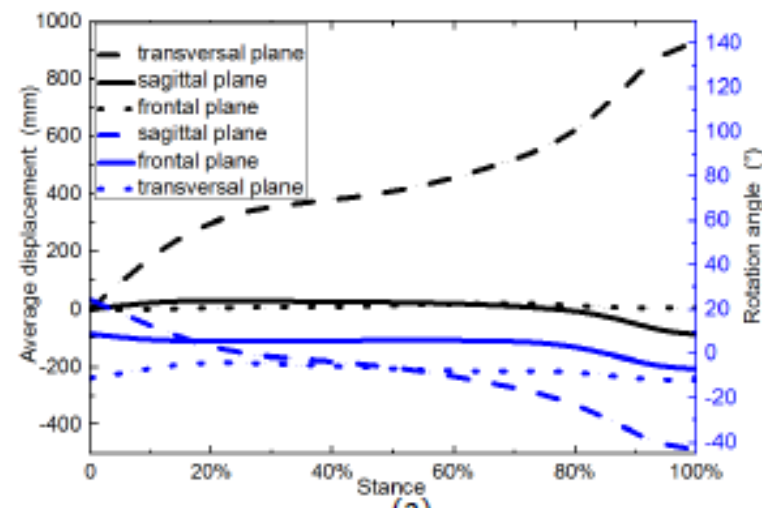

(a)

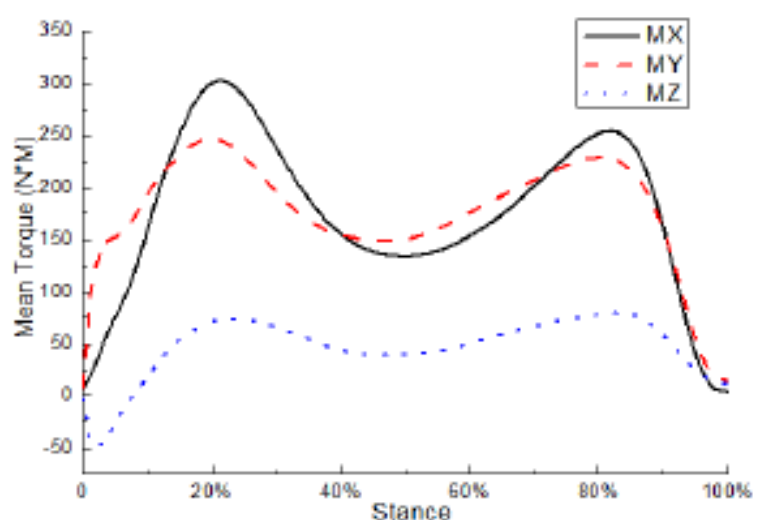

(b)

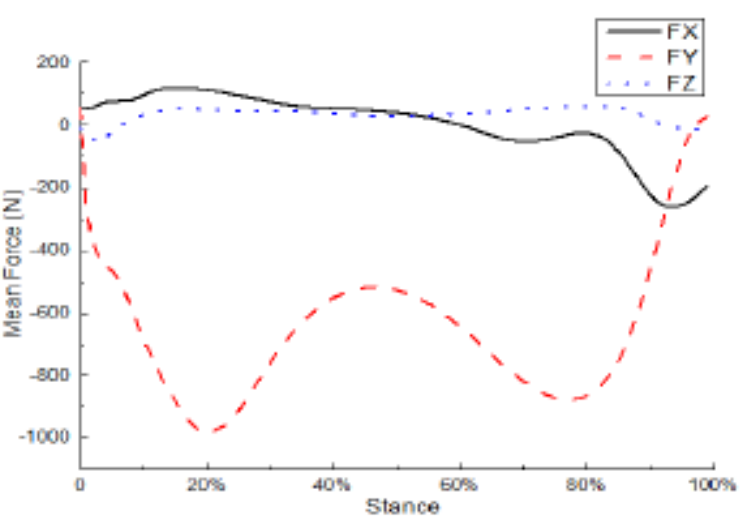

(c)

Figure.2 Simulation data at control point, (a) average displacement in three directions at control point; (b) mean forces in three directions at control point; (c) mean torques at control point

\section{Stretch Exercise Simulation}

Two stretch exercises were simulated in MSC.ADAMS software. For Achilles tendon stretch, patients are usually required to lean against a wall while the affected leg is putting behind the other foot with its toes pointing directly towards the front foot [12]. Important detail in Achilles tendon stretching is to keep the lateral leg straight and heel adhering to the ground. By bending the trunk towards the wall, Achilles tendon would be tightened. Achilles tendon stretch was performed in lab environment and 10 markers were attach to shin to track its movement using Optotrak Certus (NDI, Canada) at frequency of $100 \mathrm{~Hz}$, collecting 10 groups data, every data contained 3000 frames, which included 5 repetitions. Internal and external condyle of femur, medial and lateral malleolus of ankle joint were chosen as four calibration points to identify the spatial movement of shin. Choosing the middle point of internal and external condyle of femur as the simulation control point, the kinematic data of which was then used to drive the simulation of Achilles tendon stretch as was shown in Fig.3 


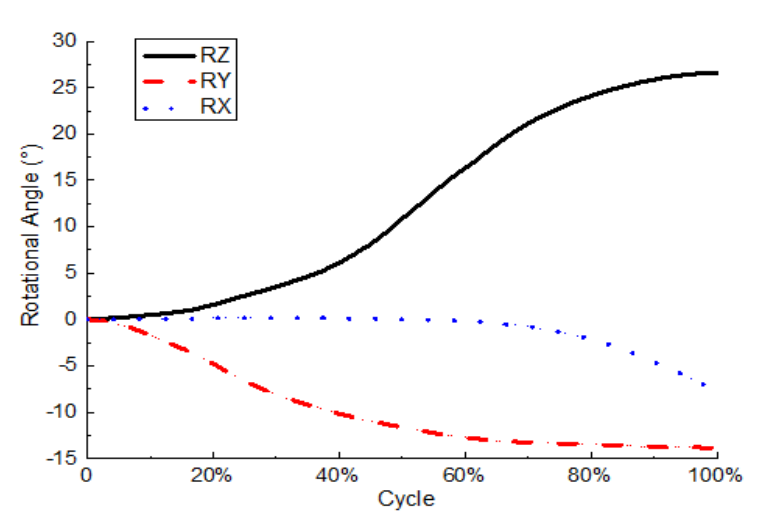

(a)

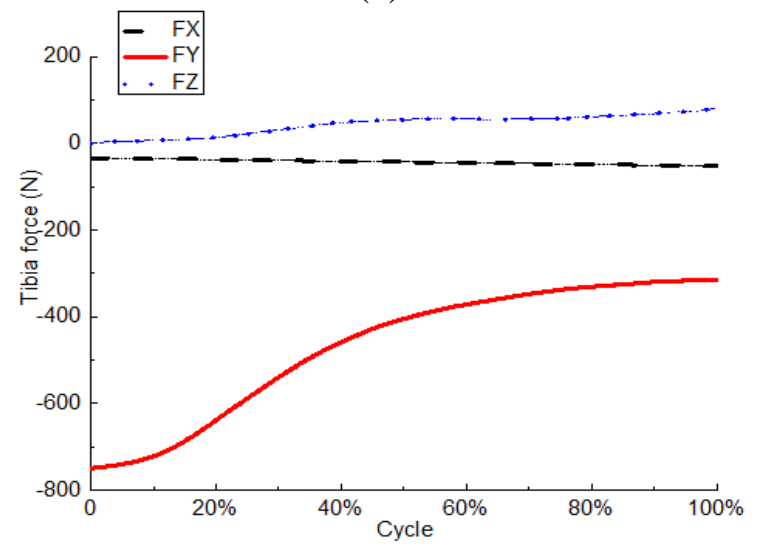

(c)

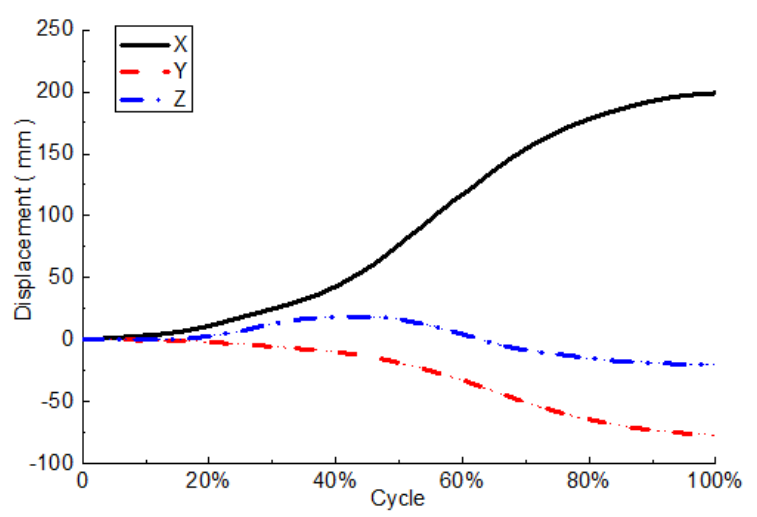

(b)

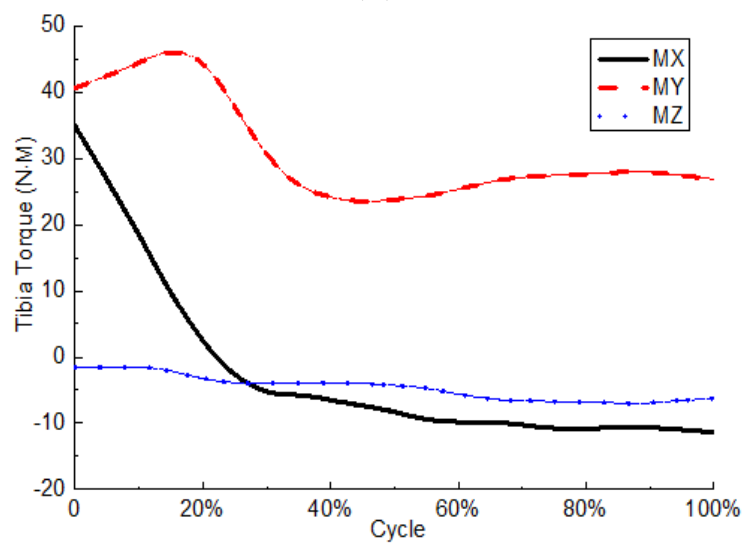

(d)

Figure.3 Experimental data of tibia in Achilles tendon stretch (a) rotational angle of tibia (b) displacement of control point (c) loading tibia force (d) loading tibia torque

In plantar fascia stretch, patients are supposed to hold their feet with one hand and bend the phalanges towards tibia using the other hand until tension in plantar fascia is felt [29]. Patients' reaction towards this stretch is different so the dorsiflexion angle of phalanges is not fixed. Based on this procedure, tibia and fibula were connected to the ground using fix joint while a rotational plate was placed beneath the forefoot in the simulation. Rotational plate has a range of motion from $0^{\circ}$ to $20^{\circ}$ with a constant angular velocity. The placing of the plate made sure that phalanges have full contact with the plate.

\section{Results}

\section{Foot Model Validation}

Ground reaction force in sagittal plane had two peaks, which occurred at $20 \%$ and $73 \%$ of stance phase and forces were 1.13 BW (body weight) and 1.2 BW. Trough was at $43 \%$ of stance phase and the force was $0.6 \mathrm{BW}$. Ground reaction force in transverse plane also had two peaks while the directions were opposite which occurred at $20 \%$ and $78 \%$ of stance phase with the values of $0.4 \mathrm{BW}$ and $0 . .32 \mathrm{BW}$ respectively. The direction of force changed at $43 \%$ of stance phase.

Ankle joint had three degree of freedom, the range of motion in sagittal plane was $12^{\circ}\left(-4^{\circ} \sim 8^{\circ}\right)$, the range of motion in frontal plane was $5^{\circ}\left(-4^{\circ} \sim 1^{\circ}\right)$, the range of motion in transversal plane was $7^{\circ}$ $\left(-3^{\circ} \sim 4^{\circ}\right)$. The other three joints had only rotation in sagittal plane. For mid-foot, the range of motion was $3^{\circ}\left(-1^{\circ} \sim 2^{\circ}\right), 4^{\circ}\left(-0.5^{\circ} \sim 3.5^{\circ}\right)$ for fore-mid foot joint and $9^{\circ}\left(-2^{\circ} \sim 7^{\circ}\right)$ for fore-foot joint.

\section{Plantar Fascia Deformation in Stretch}

Initial and terminal status of Achilles tendon stretching were presented in Fig.4. Foot was adhering to the ground while the angle between tibia and horizontal plane was $60^{\circ}$. The deformation of five rays of plantar fascia were shown in Fig.5.The 1st ray of plantar fascia had the largest deformation 
which was around $4 \mathrm{~mm}$ and the following four rays shared the same elongation pattern with smaller deformation. The average deformation during the stretch was $3.5 \mathrm{~mm}$.
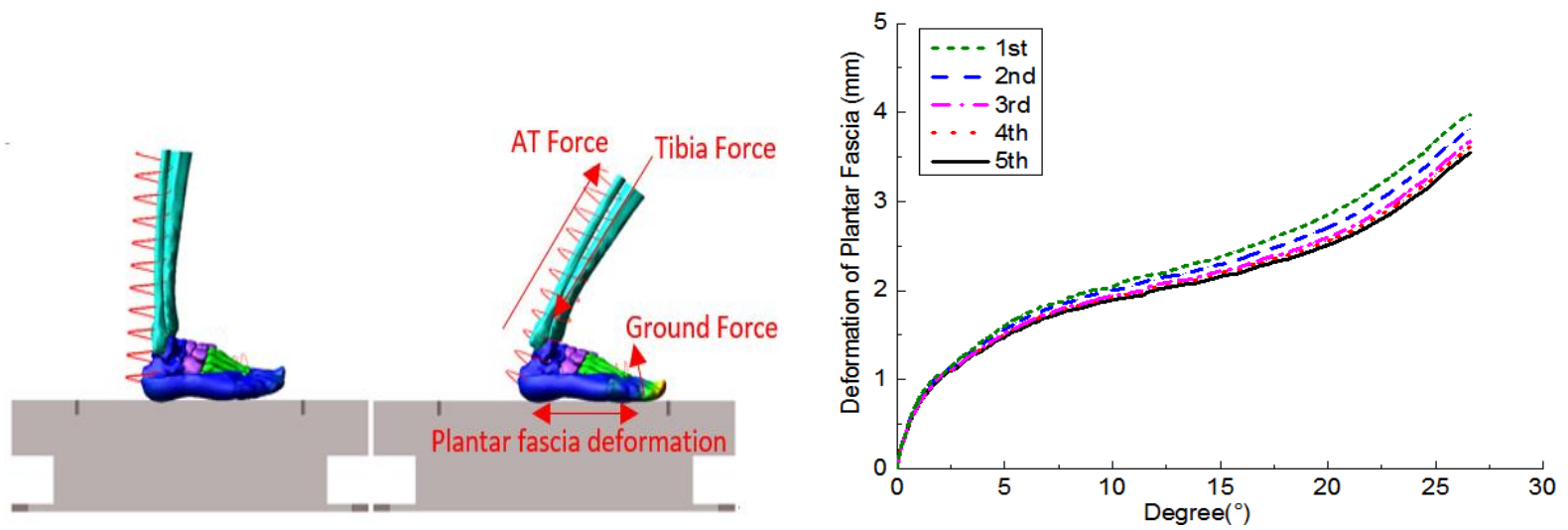

Figure 4. Achilles tendon stretch

Figure 5. Plantar fascia deformation in Achilles tendon stretch

Fig.6 shows the initial and terminal status of plantar fascia stretching. Dorsiflexion of phalange reached $20^{\circ}$ while rest of the foot model remained still. The deformation of five rays of plantar fascia was presented in Fig.6. The 1st ray of plantar fascia had deformation of $11 \mathrm{~mm}$, which was significantly larger than the other four rays. The deformation decreased from 2 nd to 4 th ray. The average deformation of the plantar fascia was $9 \mathrm{~mm}$.
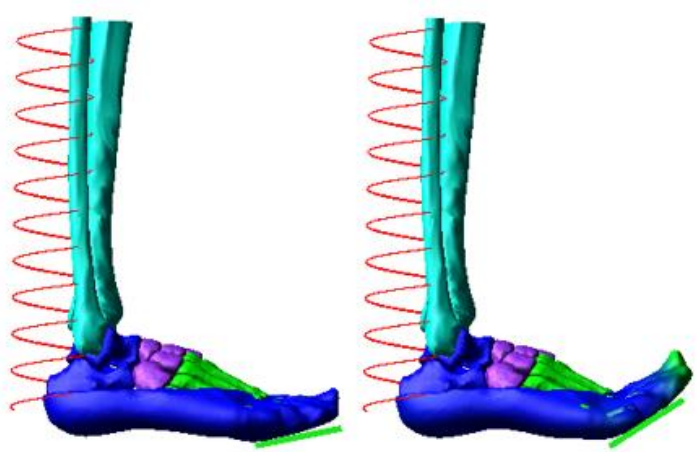

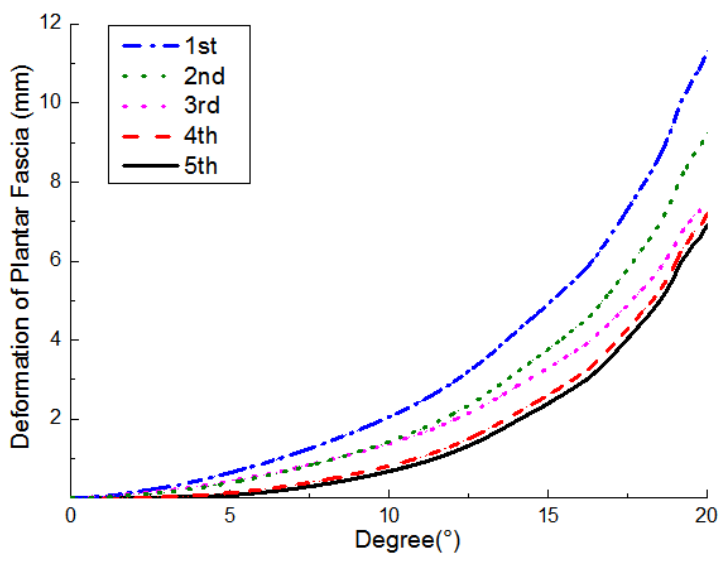

Figure 6. Plantar fascia stretch

Figure 7. Plantar fascia deformation in plantar fascia stretch

\section{Discussion and Conclusion}

Conservative methods are prior to invasive methods in plantar fasciitis treatment. Various stretching protocols have been applied to make plantar fascia regain its stiffness and relief the pain. There are clinical statistics supporting that plantar fascia stretch is more effective than Achilles tendon stretch but no theoretical evidence exists [15]. A multi-segment foot model, after validation, was used to simulate these two stretching processes, providing biomechanical analysis about the stretching methods.

Ground reaction force and range of motion in foot joints could serve as criterion in model validation. For ground reaction force, the peaks of forces in sagittal plane and frontal plane occurred at $20 \%, 43 \%$ and $73 \%$ of stance phase cycle which were consist with the finding of Lundgren, Giakas and Jung [28, 30, 31]. In Giakas's research [30], sagittal plane ground reaction force was within the range of 1.06 1.17 $\mathrm{BW}$ while the result of this paper was within 1.1 1.14 $\mathrm{BW}$. As for range of motion in joints, Lundgren [28] has measured the range of motion between talus and tibia, navicular and talus, metatarsal and cuneiform were respectively $15.3^{\circ}, 8.4^{\circ}, 5.3^{\circ}$. In MacWilliams's experiment [32], range of motion at ankle joint in sagittal plane, frontal plane and transverse plane were $13^{\circ}, 9^{\circ}, 6^{\circ}$. Motion ranges of our model in three planes were $12^{\circ}, 5^{\circ}$ and $7^{\circ}$, which were 
approximate to previous research. These two criterion indicated that our model was effective in dynamic gait simulation and can be applied in further research.

The plantar fascia deformation provided a direct insight into the differences existing between these two stretching methods. Plantar fascia stretch generated a larger deformation so the stimulation on plantar fascia was supposed to be better. Meanwhile, the 1st ray of plantar fascia both had larger deformation than the rest four rays in two stretching simulation, which complies with previous research [24].

One limit of our research is that the effect on plantar fascia brought by the tension in Achilles tendon was unable to be quantified. Plantar fasciitis patients tend to have a tight Achilles tendon which would increase the tension of plantar fascia. Our simulation provided data that plantar fascia stretch was more effective than Achilles tendon stretch on the deformation perspective. More comprehensive judging system may be required to provide overall assessment towards these two methods. Meanwhile, the characteristics of two stretching methods were identified and were then set as simulation guidance. Kinematic data from experiment was used to drive the foot model, the differences lies in subjects' habits and physical condition had not been taken as influence factors. Simulation using different material parameters would shed light on possible different reactions towards stretch treatments amongst various patients. Generally, simulation methods and results in this paper provided a quantified data in analysing the differences of two stretch treatments. Further optimization of the multi-segment model is needed assist in clinical research and improvement of physical therapies.

\section{Acknowledgement}

This work was supported by the Natural Science Foundation of China through the research project (No. 51375304).

\section{Reference}

1. C.-K. Huang, H. B. Kitaoka, K.-N. An. Foot. Ank. Inte, 14, 353(1993).

2. H. Lemont, K. M. Ammirati, N. Usen. J. Amer. Podi Med. Asso, 93, (2003)

3. W. Gibbon, G. Long. Skel. Radi, 28,21(1999)

4. R. L. Martin, T. E. Davenport, S. F. Reischl.J. Orth. Spor. Phy. Ther (2014)

5. A. MacInnes, S. C. Roberts, J. Kimpton.Foot. Ank. Inte (2015)

6. J. D. Goff, R. Crawford. Ame. Fam. Phy. 84, 676 (2011)

7. M. I. Ibrahim, R. A. Donatelli, C. Schmitz. Foot. Ank. Inte, 31,391 (2010)

8. J. T. Cheung, M. Zhang, A. K. Leun. J. Biom. 38, 1045 (2005)

9. G. Amit. J. Biom, 35, 629 (2002)

10. A. M. McMillan, K. B. Landorf, M. F. Gilheany. BMj. (2012)

11. D. H. Kamonseki, G. A. Gonçalves, C. Y. Liu. Manu. Ther, 23, 76 (2016)

12. B. F. DiGiovanni, D. A. Nawoczenski, M. E. Lintal. J. Bone.Join.Surg. Am, 85,1270 (2003)

13. A. A. Schepsis, R. E. Leach, J. Gouyca. Clin. Orth. Rese, 266, 185 (1991)

14. H.Y.K . Cheng, C.-L. Lin, H.-W. Wang. J. Biom. 41, 1937 (2008)

15. B. F. Digiovanni, D. A. Nawoczenski, D. P. Malay. J. Bone. Joint. Surg. Am. 88,1775 (2006)

16. Y. Wang, D. W.-C. Wong, M. Zhang. Ann. Biom. Eng, 44, 213 (2016)

17. X.X. Yan, D. M. Wang, J. W. Yin, J. Med. Biom. 30, 404 (2015) 
18. K. Deschamps, F. Staes, H. Bruyninckx, Gait. Post. 35, 255 (2012)

19. T. R. Jenkyn, K. Anas, A. Nichol. J. Biom. Eng. 131, 201 (2009)

20. P. Caravaggi, M. Benedetti, L. Berti, Gait. Post. 33, 133 (2011)

21. C. J. Wright, B. L. Arnold, T. G. Coffey, Gait. Post, 33108 (2011)

22. M. Millard, E. Kubica, J. McPhee, Proc. IUT, 2142 (2011)

23. M. C. Anderson, N. A. T. Brown, K. N. Bachus, Gait. Post, 3087 (2009)

24. P. Caravaggi, T. Pataky, J. Y. Goulermas. J. Exp. Bio, 212, 2491(2009)

25. P. Moreira, M. Silva, P. Flores, EUROMECH Solid Mechanics, 07 (2009)

26. A. Erdemir, P. A. Sirimamilla, J. P. Halloran, J. Biom. Eng 131, 1798 (2009)

27. J. Shin, N. Yue, C. D. Untaroiu, Ann. Biom. Eng. 40, 2519 (2012)

28. P. Lundgren, C. Nester, A. Liu, Gait. Post 28, 93 (2008)

29. D. Singh, J. Angel, G. Bentle. BMJ. 315, 172(1997)

30. G. Giakas, V. Baltzopoulos. Gait. Post, 5189 (1997)

31. Y. Jung, M. Jung, J. Ryu, Gait. Post, 845 (2016)

32. B. A. MacWilliams, M. Cowley, D. E. Nicholson, 17214 (2003) 